\title{
High quality ferromagnetic 0 and $\pi$ Josephson tunnel junctions
}

\author{
M. Weides, ${ }^{1}$ M. Kemmler, ${ }^{2}$ H. Kohlstedt,${ }^{1,3}$ A. Buzdin, ${ }^{4}$ E. Goldobin, ${ }^{2}$ D. Koelle,${ }^{2}$ and R. Kleiner ${ }^{2}$ \\ ${ }^{1}$ Center of Nanoelectronic Systems for Information Technology (CNI), \\ Research Centre Jülich, D-52425 Jülich, Germany \\ ${ }^{2}$ Physikalisches Institut - Experimentalphysik II, Universität Tübingen, \\ Auf der Morgenstelle 14, D-72076 Tübingen, Germany \\ ${ }^{3}$ Department of Material Science and Engineering and Department of Physics, University of Berkeley, California 94720, USA \\ ${ }^{4}$ Institut Universitaire de France and Condensed Matter Theory Group, CPMOH, \\ University Bordeaux 1, UMR 5798, CNRS, F-33405 Talence Cedex, France
}

\begin{abstract}
We fabricated high quality $\mathrm{Nb} / \mathrm{Al}_{2} \mathrm{O}_{3} / \mathrm{Ni}_{0.6} \mathrm{Cu}_{0.4} / \mathrm{Nb}$ superconductor-insulator-ferromagnetsuperconductor Josephson tunnel junctions. Depending on the thickness of the ferromagnetic $\mathrm{Ni}_{0.6} \mathrm{Cu}_{0.4}$ layer and on the ambient temperature, the junctions were in the 0 or $\pi$ ground state. All junctions have homogeneous interfaces showing almost perfect Fraunhofer patterns. The $\mathrm{Al}_{2} \mathrm{O}_{3}$ tunnel barrier allows to achieve rather low damping, which is desired for many experiments especially in the quantum domain. The McCumber parameter $\beta_{c}$ increases exponentially with decreasing temperature and reaches $\beta_{c} \approx 700$ at $T=2.11 \mathrm{~K}$. The critical current density in the $\pi$ state was up to $5 \mathrm{~A} / \mathrm{cm}^{2}$ at $T=2.11 \mathrm{~K}$, resulting in a Josephson penetration depth $\lambda_{J}$ as low as $160 \mu \mathrm{m}$. Experimentally determined junction parameters are well described by theory taking into account spin-flip scattering in the $\mathrm{Ni}_{0.6} \mathrm{Cu}_{0.4}$ layer and different transparencies of the interfaces.
\end{abstract}

PACS numbers: 74.25 .Fy $74.45 .+\mathrm{c} 74.50 .+\mathrm{r}, 74.70 . \mathrm{cn}$

The realization of solid state qubits attracts considerable interest. Josephson junctions (JJs) are used to realize charge [1, phase 2] or flux 3] qubits. For the "quiet" flux qubit [4], which is self-biased and well decoupled from the environment, one needs to use high quality $\pi$ JJs with high resistance (to avoid decoherence) and reasonably high critical current density $j_{c}$ (to have the Josephson energy $E_{J} \gg k_{B} T$ for junction sizes of few microns or below). High $j_{c}$ is also required to keep the Josephson plasma frequency $\omega_{p} \propto \sqrt{j_{c}}$, which plays the role of an attempt frequency in the quantum tunneling problem, on the level of a few GHz.

The concept of $\pi$ JJs was introduced long ago [5, 6], but only recently superconductor-ferromagnet-superconductor (SFS) $\pi$ JJs were realized [7, [8]. Unfortunately SFS $\pi$ JJs are highly overdamped and cannot be used for applications where low dissipation is required. The obvious way to decrease damping is to make a SFS-like tunnel junction, i.e. a superconductor-insulator-ferromagnet-superconductor (SIFS) junction. Due to the presence of the tunnel barrier the critical current $I_{c}$ in SIFS is lower than in SFS, but both the resistance $R$ (at $I \gtrsim I_{c}$ ) and the $I_{c} R$ product are much higher. Moreover, the value of $I_{c}$ and $R$ can be tuned by changing the thickness $d_{I}$ of the insulator (tunnel barrier).

A set of SIFS JJs with different thickness $d_{F}$ of the F-layer were recently fabricated and JJs with both 0 and $\pi$ ground states were observed depending on $d_{F}[\underline{9}$. Although, in the $\pi$ state the specific resistance of the barrier was high $\left(\rho \sim 3 \mathrm{~m} \Omega \cdot \mathrm{cm}^{2}\right), j_{c}$ was below $7 \mathrm{~mA} / \mathrm{cm}^{2}$ at $1.5 \mathrm{~K}$, resulting in an $I_{c} R$ product below $20 \mu \mathrm{V}$, as can be estimated from the data in Ref. 9 .

In this letter we report on fabrication and characterization of high quality $\mathrm{Nb} / \mathrm{AlO}_{x} / \mathrm{Ni}_{y} \mathrm{Cu}_{1-y} / \mathrm{Nb}$ JJs with different $d_{F}$ having as high as possible $j_{c}$ and $I_{c} R$ values.
In the $\pi$ state we reached $j_{c}$ up to $5 \mathrm{~A} / \mathrm{cm}^{2}$ at $T=2.11 \mathrm{~K}$ and maximum $I_{c} R$ values $\approx 400 \mu \mathrm{V}$. SIFS and reference SIS JJs were fabricated in-situ by magnetron sputtering and patterned using optical lithography and (reactive) dry-etching [10]. On thermally oxidized $\mathrm{Si}$ wafers we deposited $120 \mathrm{~nm} \mathrm{Nb}$ and $5 \mathrm{~nm} \mathrm{Al}$. To form the $\mathrm{Al}_{2} \mathrm{O}_{3}$ barrier (which should be as thin as possible, but without pinholes) we oxidized at 0.015 or at 50 mbar to have $j_{c}^{(1)} \approx 4.0 \mathrm{kA} / \mathrm{cm}^{2}$ (wafer 1 ) and $j_{c}^{(2)} \approx 0.19 \mathrm{kA} / \mathrm{cm}^{2}$ (wafer 2) for reference SIS JJs. For reference SIS JJs on wafer 1 the $I_{c} R$ product was $1.55 \mathrm{mV}$.

To control the properties of SIFS JJs the thickness and the roughness of the F-layer should be controlled on a sub-nm scale. To provide uniform growth of the F-layer, a $2 \mathrm{~nm} \mathrm{Cu}$ interlayer was deposited between I-layer and F-layer. As F-layer we used diluted $\mathrm{Ni}_{0.6} \mathrm{Cu}_{0.4}$, followed by a $40 \mathrm{~nm} \mathrm{Nb}$ cap-layer. To produce JJs with different $d_{F}$ in a single run, during sputtering of the F-layer, the substrate and sputter target were shifted about half the substrate length producing a wedge-like F-layer with $d_{F}$ from 1 to $15 \mathrm{~nm}$ across the 4" wafer. All other layers had uniform thicknesses. The SIFS junctions had a squared shape with an area of $100 \times 100 \mu \mathrm{m}^{2}$.

We have used diluted $\mathrm{Ni}_{y} \mathrm{Cu}_{1-y}$ alloy rather than pure $\mathrm{Ni}$ to have suitable $d_{F}$ (much larger than roughness) for the $\pi$ state. In very diluted alloy with $y \leq 0.53$ strong spin-flip scattering [11] and Ni cluster formation are observed 12, 13. To avoid this magnetic inhomogeneity we have used $y=0.6$, as confirmed by Rutherford backscattering spectroscopy. The Curie temperature $T_{\mathrm{C}} \sim 225 \mathrm{~K}$ was determined by SQUID magnetometry and anisotropic Hall measurements on bare $\mathrm{Ni}_{0.6} \mathrm{Cu}_{0.4}$ films. Both $T_{\mathrm{C}}$ and resistivity $\rho_{F}(10 \mathrm{~K})=54 \mu \Omega \cdot \mathrm{cm}$ are in good agreement with the literature 14, 15. The magnetization of such thin $\mathrm{Ni}_{0.6} \mathrm{Cu}_{0.4}$ films is in-plane. 
Interpolation of the magnetic moment $\mu$ from published data [1], 16, 17, 18] yields $\mu=0.15 \mu_{B}$ per atom for our $\mathrm{Ni}_{0.6} \mathrm{Cu}_{0.4}$ alloy.

Following Ref. 19 one can derive that at $T \lesssim T_{c}$

$$
I_{c}\left(d_{F}\right) \sim \frac{1}{\gamma_{B 2}} \exp \left(\frac{-d_{F}}{\xi_{F 1}}\right) \cos \left(\frac{d_{F}-d_{F}^{\text {dead }}}{\xi_{F 2}}\right),
$$

where $\xi_{F 1, F 2}=\xi_{F} / \sqrt{\sqrt{1+\alpha^{2}} \pm \alpha}$ are the decay and oscillation lengths of order parameter 20$], \xi_{F}=$ $\sqrt{\hbar D / E_{\text {ex }}}$ is the decay/oscillation length without spinflip scattering [19], $E_{\mathrm{ex}}$ is the exchange energy, $\alpha=$ $1 /\left(\tau_{s} E_{\mathrm{ex}}\right), \tau_{s}$ is the inelastic magnetic scattering time 21] and $\gamma_{B 2}$ is the transparency parameter of the SIF part treated like a single interface. $d_{F}^{\text {dead }}$ is the magnetic dead layer thickness. Eq. (1) is derived assuming that the interfaces are not spin active, cf. 22], short decay length $\xi_{F 1}<d_{F}, \xi_{F 1} \ll \xi_{F 2}$ and FS interface transparency parameter $\gamma_{B 1}=0\left(\gamma_{B 1} \ll \gamma_{B 2}\right)$. In comparison with 23] Eq. (1) takes into account magnetic impurity scattering which enters via $\tau_{s}$. Since $\xi_{F 2}$ weakly depends on temperature $T$, the $0-\pi$ crossover can be observed by changing $T$.

The spread in $j_{c}$ among SIFS JJs with the same $d_{F}$ is about $2 \%[10]$. The $I_{c}\left(d_{F}\right)$ dependence of our SIFS JJs is clearly non monotonic as shown in Fig. 1] We argue that the minimum of $I_{c}\left(d_{F}\right)$ at $d_{F} \approx 5.21 \mathrm{~nm}$ corresponds to 0 to $\pi$ crossover. To rule out the possibility of $0-\pi$ crossover at smaller $d_{F}$ we have investigated $I_{c}\left(d_{F}\right)$ down to $d_{F}=2 \mathrm{~nm}$ and did not observe any decrease or oscillation of $I_{c}\left(d_{F}\right)$. In Fig. 11 we show only data for "low" $j_{c}$ JJs $\left(L<2 \lambda_{J}\right)$ that we can treat as short JJs to fit experimental $I_{c}\left(d_{F}\right)$ using Eq. (1). Due to a finite dead magnetic layer the change of phase takes place in an effectively reduced F-layer thickness. By fitting $I_{c}\left(d_{F}\right)$ for wafer 1 using Eq. (1), we estimated $\xi_{F 1}=0.78 \mathrm{~nm}$,

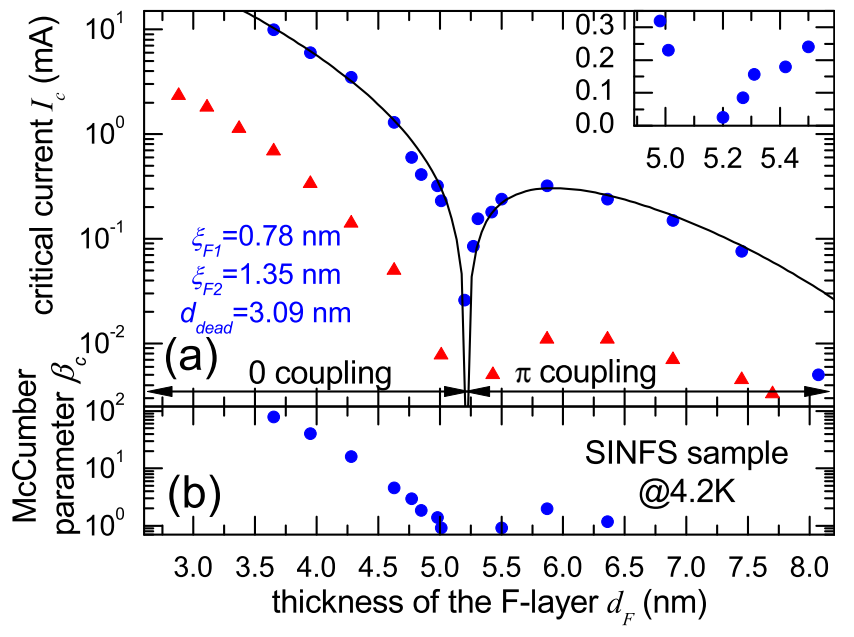

FIG. 1: (Color online) $I_{c}\left(d_{F}\right)(\mathrm{a})$ and $\beta_{c}\left(d_{F}\right)$ (b) dependence (circles: wafer 1, triangles: wafer 2 ) and fitting curve for wafer 1. Inset shows magnification of 0 to $\pi$ transition region for the wafer 1 on a linear scale.

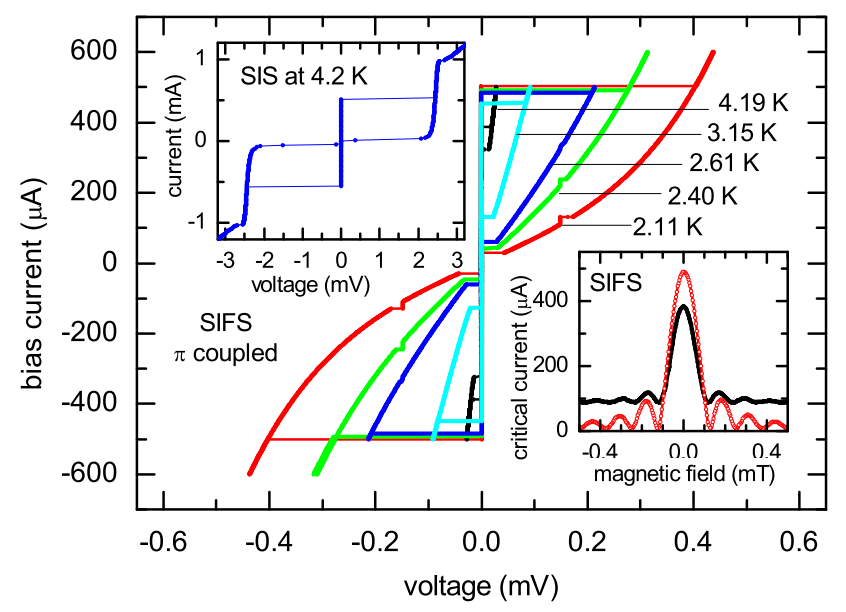

FIG. 2: (Color online) IVCs of $\pi$ SIFS JJ $\left(d_{F}=5.87 \mathrm{~nm}\right)$ at different $T$. Insets show: IVC of SIS JJ at $T=4.2 \mathrm{~K}$ (top) and $I_{c}(H)$ of SIFS JJ at $T=4.2$ and $2.11 \mathrm{~K}$

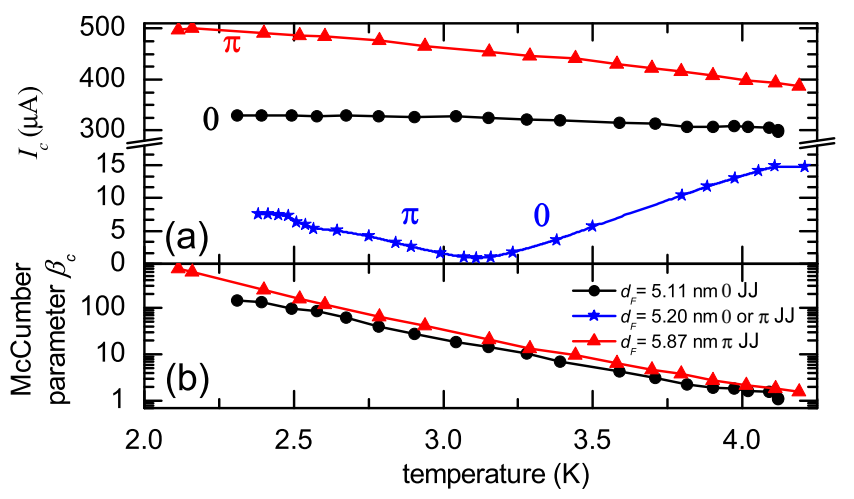

FIG. 3: (Color online) $I_{c}(T)$ (a) and $\beta_{c}(T)$ (b) dependence of 0 and $\pi$ SIFS JJs.

$\xi_{F 2}=1.35 \mathrm{~nm}$ and $d_{F}^{\text {dead }}=3.09 \mathrm{~nm}$. As we see, the inelastic magnetic scattering is strong $\left(\xi_{F 1}<\xi_{F 2}\right)$ and the decay length $\xi_{F 1} \ll d_{F}$, thus Eq. (1) is applicable. Also, the found value of $d_{F}^{\text {dead }}$ supports our claim that we observed 0 to $\pi$ rather than $\pi$ to 0 crossover. According to Eq. (1) the coupling changes from 0 to $\pi$ at the crossover thickness $d_{F}^{0-\pi}=\frac{\pi}{2} \xi_{F 2}+d_{F}^{\text {dead }}=5.21 \mathrm{~nm}$, the shape of the $I_{c}\left(d_{F}\right)$ curve does not change with the thickness of the insulator, but the amplitude of $I_{c}\left(d_{F}\right)$ is proportional to the reciprocal transparency parameter $\gamma_{B 2}^{-1}$. In the interval of $d_{F}$ from 0 (for SIS) to $9 \mathrm{~nm}$ the value of $j_{c}$ at $4.2 \mathrm{~K}$ changes over five orders of magnitude from $4 \mathrm{kA} / \mathrm{cm}^{2}$ to below $0.05 \mathrm{~A} / \mathrm{cm}^{2}$ (wafer 1 ).

The maximum $j_{c}$ in the $\pi$ state is $3.8 \mathrm{~A} / \mathrm{cm}^{2}$ (wafer 1 ) and $j_{c}(\pi)=90 \mathrm{~mA} / \mathrm{cm}^{2}$ (wafer 2 ) at $T=4.2 \mathrm{~K}$. This gives $\lambda_{J} \gtrsim 190 \mu \mathrm{m}$, which can be easily increased by increasing $d_{I}$. Further decrease of $\lambda_{J}$ by lowering $d_{I}$ is limited by the appearance of microshorts in the barrier.

For comparison, in Ref. 21 SFS JJs were fabricated using the weaker ferromagnet $\mathrm{Ni}_{0.53} \mathrm{Cu}_{0.47}\left(T_{\mathrm{C}}=60 \mathrm{~K}\right)$. Although the spin-flip scattering was also taken into ac- 
count, the high interface transparencies $\left(\gamma_{B 1}=0.52\right)$ lead to a different $I_{c}\left(d_{F}\right)$ dependence than Eq. (1) predicts. Also, the lower $E_{\mathrm{ex}}$ lead to larger $\xi_{F 1}=1.24 \mathrm{~nm}$ and $\xi_{F 2}=3.73 \mathrm{~nm}$. The magnetic dead layer was 1.4 times larger than in our system.

Fig. 1(b) shows the dependence of the McCumber parameter $\beta_{c}\left(d_{F}\right)$, which was estimated from the values of $I_{c}$ and $I_{r}$ (return current), at $T=4.2 \mathrm{~K}$ for wafer 1 . The capacitance $C \approx 800 \mathrm{pF}$, determined from the Fiske step spacing of $73 \mu \mathrm{V}$ is nearly independent from $d_{F}$, but depends on $d_{I}$. Near the $0-\pi$ crossover and for large $d_{F}$ the value of $I_{c}$ is very low and the junctions become overdamped $\left(\beta_{c}<0.7\right)$. For $\pi$ JJs with $d_{F}$ near the maxima of the $I_{c}\left(d_{F}\right)$ curve a hysteresis appears on the $I$ - $V$ characteristic (IVC).

The IVCs and $I_{c}(H)$ patterns (voltage criterion $5 \mu \mathrm{V}$ ) for a SIFS $\pi$ JJ with highest $I_{c}$ are shown in Fig. 2 c.f. the IVC of the SIS JJs shown in the inset. Theoretically, at lower temperature the quasiparticle current decreases and the gap appears at higher voltages. In experiment, due to heating effects at high bias currents, part of the sample became normal before we were able to reach the gap voltage. At $T \leq 2.61 \mathrm{~K}$ the first zero field step at $149 \mu \mathrm{V}$ is visible on the IVC.

The energy dependence of the density of states in $\mathrm{Al}$, $\mathrm{Cu}$ and $\mathrm{NiCu}$ are not exactly BCS-like and $I_{c}(T)$ for SIFS JJs should show a more linear behavior 24 than originally found by Ambegaokar-Baratoff [25]. Variation of $T$ modifies $\xi_{F 1}$ and $\xi_{F 2}$ and can even change the ground state [7, 11]. Since $E_{\text {ex }}$ of $\mathrm{Ni}_{0.6} \mathrm{Cu}_{0.4}$ is relatively large, a change of $T$ affects our JJs much less than in previous work on the stronger diluted $\mathrm{NiCu}$ alloys [7, 11]. The $I_{c}(T)$ dependences for three JJs from wafer 1 are shown in Fig. 31 (a). At $d_{F}=5.11 \mathrm{~nm}$ the JJ is 0 coupled, but we attribute the nearly constant $I_{c}$ below $3.5 \mathrm{~K}$ to the interplay between increasing Cooper pair density and decreasing oscillation length $\xi_{F 2}(T)$. The JJ with $d_{F}=5.20 \mathrm{~nm}$ is 0 coupled at $T=4.2 \mathrm{~K}$, but changes coupling to $\pi$ be- low $3.11 \mathrm{~K}$. During the $0-\pi$ transition its critical current is not vanishing completely $\left(I_{c}^{\min } \approx 0.8 \mu \mathrm{A}\right)$ either due to roughness of the ferromagnet or a prominent $\sin (2 \phi)$ component in the current-phase relation 26, 27], which can appear intrinsically or again due to roughness [28, 29]. At the crossover temperature $T_{x}=3.11 \mathrm{~K}, I_{c}(H)$ can still be traced through several minima, so the large scale roughness must be small. The $d_{F}=5.87 \mathrm{~nm}$ JJ (also shown in Fig. 2) exhibits the highest critical current among $\pi$ JJs $\left(j_{c}=5 \mathrm{~A} / \mathrm{cm}^{2}\right.$ at $\left.2.11 \mathrm{~K}\right)$. Up to now the corresponding $\lambda_{J}=160 \mu \mathrm{m}$ is the smallest achieved for SIFS JJs. Fig. [3(b) shows $\beta_{c}(T)$ for the same JJs. $\beta_{c}(T)$ increases exponentially below $4 \mathrm{~K}$ for both 0 and $\pi$ JJs, indicating very weak Cooper pair breaking in the F-layer for these temperatures. The $\beta_{c}$ of the always overdamped JJ with $d_{F}=5.20 \mathrm{~nm}$ was not estimated.

In summary, we have fabricated and investigated SIFS Josephson junctions with $\mathrm{Ni}_{0.6} \mathrm{Cu}_{0.4}$ F-layer and thin $\mathrm{Al}_{2} \mathrm{O}_{3}$ tunnel barriers. The critical current $I_{c}$ changes sign as a function of the F-layer thickness $d_{F}$ in accordance with theory, exhibiting regions with 0 and $\pi$ ground states. For $d_{F}$ near the 0 to $\pi$ crossover the ground state can be controlled by changing the temperature. Our SIFS $\pi$ junctions show critical current densities $j_{c}$ up to $5 \mathrm{~A} / \mathrm{cm}^{2}$ at $T=2.11 \mathrm{~K}$ and $I_{c} R$ products about $400 \mu \mathrm{V}$. The achieved $\pi$ junction's Josephson penetration depth $\lambda_{J}$ as low as $160 \mu \mathrm{m}$ at $2.11 \mathrm{~K}$ allows to fabricate long Josephson 0- $\pi$ junctions of reasonable size and study half integer flux quanta (semifluxons) that appear at the $0-\pi$ boundaries $30,31,32$ and have a size $\sim \lambda_{J}$. Reasonable $\lambda_{J}$ and low damping in such $0-\pi$ junctions may lead to useful classical 33, 34] or quantum 35, 36, 37] circuits based on semifluxons.

We thank B. Hermanns for help with fabrication and V. Ryazanov for fruitful discussions. This work is supported by ESF program PiShift and by the DFG projects GO-1106/1 and SFB/TR 21.
[1] Y. Nakamura, Y. A. Pashkin, and J. S. Tsai, Nature 398, 786 (1999).

[2] J. M. Martinis, S. Nam, J. Aumentado, and C. Urbina, Phys. Rev. Lett. 89, 117901 (2002).

[3] J. E. Mooij, T. P. Orlando, L. Levitov, L. Tian, C. H. van der Wal, and S. Lloyd, Science 285, 1036 (1999).

[4] L. B. Ioffe, V. B. Geshkenbein, M. V. Feigel'man, A. L. Faucheère, and G. Blatter, Nature 398, 679 (1999).

[5] V. K. L. Bulaevskii and A. Sobyanin, JETP Lett. 25, 7 (1977).

[6] A. Buzdin and M. Kupriyanov, JETP Lett 53, 321 (1991).

[7] V. V. Ryazanov, V. A. Oboznov, A. Y. Rusanov, A. V. Veretennikov, A. A. Golubov, and J. Aarts, Phys. Rev. Lett. 86, 2427 (2001).

[8] Y. Blum, A. Tsukernik, M. Karpovski, and A. Palevski, Phys. Rev. Lett. 89, 187004 (2002).

[9] T. Kontos, M. Aprili, J. Lesueur, F. Genêt, B. Stephani- dis, and R. Boursier, Phys. Rev. Lett. 89, 137007 (2002).

[10] M. Weides, K. Tillmann, and H. Kohlstedt, Physica C 437-438, 349 (2006).

[11] H. Sellier, C. Baraduc, F. Lefloch, and R. Calemczuk, Phys. Rev. B 68, 054531 (2003).

[12] K. Levin and D. L. Mills, Phys. Rev. B 9, 2354 (1974).

[13] R. W. Houghton, M. P. Sarachik, and J. S. Kouvel, Phys. Rev. Lett. 25, 238 (1970).

[14] A. T. Hindmarch, C. H. Marrows, and B. J. Hickey, Phys. Rev. B 72, 100401 (2005).

[15] J. C. Ododo and B. R. Coles, J. Phys. F: Met. Phys. 7, 2393 (1977).

[16] F. Brouers, A. V. Vedyayev, and M. Giorgino, Phys. Rev. B 7, 380 (1973).

[17] V. V. Ryazanov, V. A. Oboznov, A. S. Prokofiev, V. Bolginov, and A.K.Feofanov, J. Low. Temp. Phys. 136, 385 (2004).

[18] A. Rusanov, R. Boogaard, M. Hesselberth, H. Seiler, and 
J. Aarts, Physica C 369, 300 (2002).

[19] A. I. Buzdin, Rev. Mod. Phys. 77, 935 (2005).

[20] E. A. Demler, G. B. Arnold, and M. R. Beasley, Phys. Rev. B 55, 15174 (1997).

[21] V. A. Oboznov, V. V. Bol'ginov, A. K. Feofanov, V. V. Ryazanov, and A. I. Buzdin, Phys. Rev. Lett. 96, 197003 (2006).

[22] A. Cottet and W. Belzig, Phys. Rev. B 72, 180503 (2005).

[23] A. Buzdin and I. Baladie, Phys. Rev. B 67, 184519 (2003).

[24] N. L. Rowell and H. J. T. Smith, Canadian J. Phys. 54, 223 (1976).

[25] V. Ambegaokar and A. Baratoff, Phys. Rev. Lett. 10, 486 (1963).

[26] A. Buzdin, Phys. Rev. B 72, 100501 (2005).

[27] M. Houzet, V. Vinokur, and F. Pistolesi, Phys. Rev. B 72, 220506 (2005).

[28] R. G. Mints, I. Papiashvili, J. R. Kirtley, H. Hilgenkamp, G. Hammerl, and J. Mannhart, Phys. Rev. Lett. 89, 067004 (2002).
[29] A. Buzdin and A. E. Koshelev, Phys. Rev. B 67, 220504 (2003).

[30] J. R. Kirtley, K. A. Moler, and D. J. Scalapino, Phys. Rev. B 56, 886 (1997).

[31] E. Goldobin, D. Koelle, and R. Kleiner, Phys. Rev. B 67, 224515 (2003).

[32] H. Susanto, S. A. van Gils, T. P. P. Visser, Ariando, H.-J. H. Smilde, and H. Hilgenkamp, Phys. Rev. B 68, 104501 (2003).

[33] N. Lazarides, Phys. Rev. B 69, 212501 (2004).

[34] H. Susanto, E. Goldobin, D. Koelle, R. Kleiner, and S. A. van Gils, Phys. Rev. B 71, 174510 (2005).

[35] T. Kato and M. Imada, J. Phys. Soc. Jpn. 66, 1445 (1997).

[36] T. Koyama, M. Machida, M. Kato, and T. Ishida, Physica C 426-431, 1561 (2005).

[37] E. Goldobin, K. Vogel, O. Crasser, R. Walser, W. P. Schleich, D. Koelle, and R. Kleiner, Phys. Rev. B 72, 054527 (2005). 